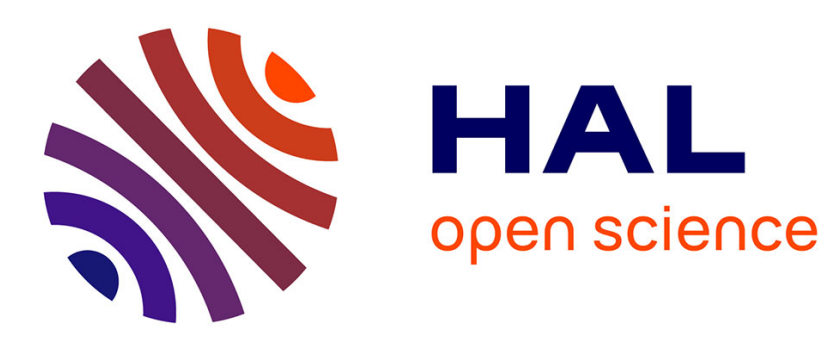

\title{
Relation entre composition paramètre de maille et bande interdite des composés Pb1-xCdxTe
}

\author{
Y. Kulvitit, S. Rolland, R. Granger, C.M. Pelletier
}

\section{To cite this version:}

Y. Kulvitit, S. Rolland, R. Granger, C.M. Pelletier. Relation entre composition paramètre de maille et bande interdite des composés Pb1-xCdxTe. Revue de Physique Appliquée, 1980, 15 (10), pp.15011504. 10.1051/rphysap:0198000150100150100 . jpa-00244874

\section{HAL Id: jpa-00244874 https://hal.science/jpa-00244874}

Submitted on 1 Jan 1980

HAL is a multi-disciplinary open access archive for the deposit and dissemination of scientific research documents, whether they are published or not. The documents may come from teaching and research institutions in France or abroad, or from public or private research centers.
L'archive ouverte pluridisciplinaire HAL, est destinée au dépôt et à la diffusion de documents scientifiques de niveau recherche, publiés ou non, émanant des établissements d'enseignement et de recherche français ou étrangers, des laboratoires publics ou privés. 


\title{
Relation entre composition paramètre de maille et bande interdite des composés $\mathrm{Pb}_{1-x} \mathrm{Cd}_{x} \mathrm{Te}$
}

\author{
Y. Kulvitit, S. Rolland, R. Granger et C. M. Pelletier \\ I.N.S.A., Laboratoire de Physique des Solides, 35043 Rennes Cedex, France
}

(Reçu le 2 avril 1980, révisé le 23 juin 1980, accepté le 4 juillet 1980)

\begin{abstract}
Résumé. - Les relations entre les valeurs expérimentales de composition en cadmium $x$, de paramètre de maille $a$ et de largeur de bande interdite $E_{\mathrm{g}}$, des composés $\mathrm{Pb}_{1-x} \mathrm{Cd}_{x} \mathrm{Te}$, sont présentées pour $x<0,15$. Ces résultats sont quelque peu différents de ceux déjà présentés. La différence est expliquée par la présence d'une plus grande quantité de cadmium précipitée sous forme de CdTe dans les matériaux obtenus antérieurement. On montre que la présence d'une fraction de cadmium précipitée de quelques \% ne peut être mise en évidence par mesure de paramètre de maille et de masse volumique.
\end{abstract}

\begin{abstract}
Experimental results about lattice constant and band gap versus composition $x$ of $\mathrm{Pb}_{1-x} \mathrm{Cd}_{x} \mathrm{Te}$ compounds are reported here. These results are somewhat different from those already published. This discrepancy is explained by the presence of CdTe precipitates in the materials used previously. It's pointed out that density and lattice constant measurements cannot show the existence of several percent of precipitated CdTe.
\end{abstract}

1. Introduction. - Alors que les principales propriétés des composés binaires II-VI et IV-VI ont été largement étudiées, peu de données existent concernant les pseudo binaires obtenus à partir des solutions solides de ces deux sortes de composés. Seuls quelques résultats concernant les paramètres physiques et la structure de bande des solutions solides $\mathrm{Pb}_{1-x} \mathrm{Cd}_{x} \mathrm{Te}$ sont connus. D'après Rosenberg et al. [1] $x$ ne peut dépasser 0,2 tandis que les matériaux sur lesquels ont été réalisées des mesures de paramètre de maille, de largeur de bande interdite et d'effet de transport [1-6] avaient une concentration en cadmium ne dépassant pas la valeur $x=0,15$. Les mesures de concentration en cadmium ne permettent pas d'affirmer qu'il provient uniquement de la solution solide. En particulier, il n'est pas exclu qu'une partie précipite sous forme de CdTe lors de l'élaboration compte tenu de la solubilité rétrograde de $\mathrm{CdTe}$ dans $\mathrm{PbTe}$; la valeur réelle de $x$ serait alors mal connue et la relation entre les paramètres physiques et la composition $x$ serait erronée. Nos résultats montrent que, si la loi de variation de la largeur de bande interdite en fonction du paramètre de maille est bien la même que celle tirée des résultats de Rosenberg et Nikolic $[1,3]$ nos valeurs de paramètre de maille et de largeur de bande interdite correspondent à des concentrations en cadmium plus faibles. Nous montrerons que la fraction précipitée sous forme de CdTe ne peut être mise en évidence par des mesures de masse volumique standards. Néan- moins nos résultats laissent à penser que nous sommes parvenus à limiter davantage cette précipitation.

\section{Elaboration des matériaux et méthodes d'analyse.} - Les lingots de solution solide $\mathrm{Pb}_{1-x} \mathrm{Cd}_{x} \mathrm{Te}$ pour $x<0,2$ [1] ont été obtenus par synthèse directe à partir des composés binaires $\mathrm{PbTe}$ et $\mathrm{CdTe}$ [7]. Après un temps d'homogénéisation en phase liquide de 24 heures les lingots ont été tirés par la méthode de Bridgman à la vitesse de $1 \mathrm{~mm} /$ heure puis trempés à l'air. Les clichés de diffraction $\mathrm{X}$ indiquent un matériau ayant la même structure (type $\mathrm{NaCl}$ ) que $\mathrm{PbTe}$ croissant suivant la direction (100), les cristallites ayant des orientations légèrement différentes autour de (100).

La composition des matériaux obtenus a été déterminée par analyse à la microsonde électronique. Des tests effectués sur trois microsondes ont donné des résultats identiques à $1 \%$ près [8]. Pour certains échantillons la teneur en cadmium a été obtenue par absorption atomique et a donné les mêmes résultats que ceux de la sonde électronique.

Les paramètres de maille sont déduits de l'analyse de clichés de Debye-Scherrer sur des poudres qui ont été recuites pour éviter l'élargissement des raies dû à l'écrouissage pendant l'opération de broyage. Les poudres ainsi recuites sont trempées dans une solution de $\mathrm{CaCl}_{2}$ à $-40^{\circ} \mathrm{C}$. Le paramètre de maille déduit de clichés de Debye-Scherrer est connu avec une erreur estimée à $10^{-3} \AA$. 
La valeur, à $300 \mathrm{~K}$ de la largeur de bande interdite $E_{\mathrm{g}}$ est déduite (cf. Fig. 1) de la variation, en fonction de l'énergie des photons, du coefficient d'absorption $\alpha(h v) \propto-\ln \frac{I(h v)}{I_{0}(h v)}$ où $I(h v)$ et $I_{0}(h v)$ sont les intensités lumineuses avec et sans le matériau étudié. Les échantillons sont des lames de $100 \mu \mathrm{m}$ d'épaisseur polies optiquement tirées de lingots polycristallins. Cette épaisseur a été choisie afin de limiter l'absorption due aux porteurs libres.

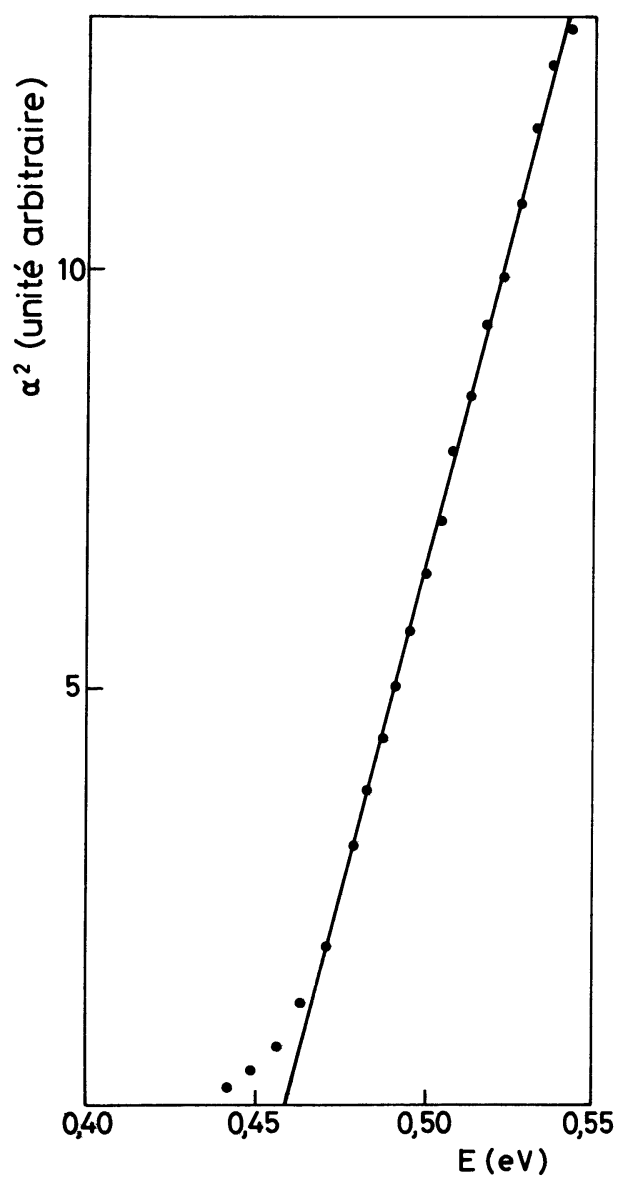

Fig. 1. - Variation du carré du coefficient d'absorption d'un échantillon ayant $x=0,07$ en fonction de l'énergie des photons.

[Square of the absorption coefficient versus photon energy for a sample with $x=0.07$.]

3. Résultats. - La figure 2 présente la variation du paramètre de maille $a$ avec $x$; cette variation est assez bien décrite par la relation :

$$
a(\AA)=6,459-0,4 x=a_{\mathrm{PbTe}}-0,4 x .
$$

Sur la même figure sont présentés les résultats de Rosenberg et al. [1] pour lesquels le paramètre de maille varie avec $x$ suivant la relation :

$$
a(\AA)=6,459-0,3 x .
$$

Il n'a pas été possible d'obtenir de solution solide

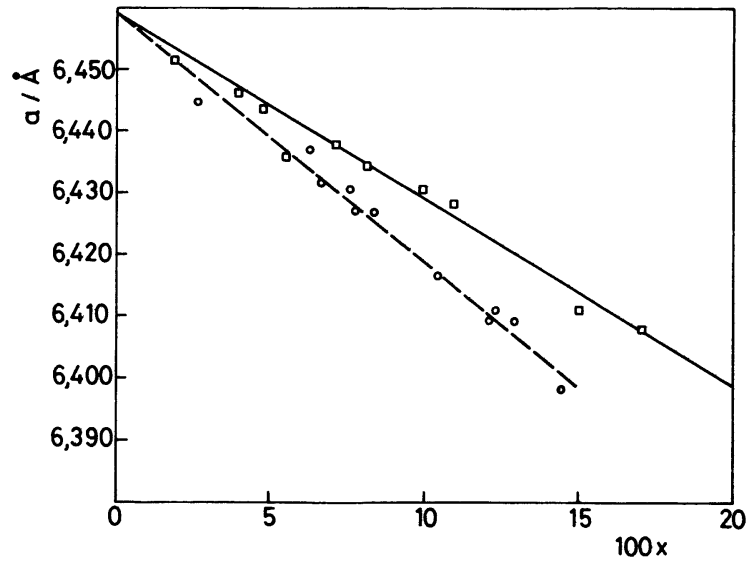

Fig. 2. - Paramètre de maille $a$ en fonction de $x$ des composés $\mathrm{Pb}_{1-x} \mathrm{Cd}_{x} \mathrm{Te}: O$ présents résultats; $\square$ résultats de Rosenberg et al. [1].

[Lattice constant variation versus $x$ for $\mathrm{Pb}_{1-x} \mathrm{Cd}_{x} \mathrm{Te}$ alloys : $\mathrm{O}$ our results; $\square$ The results of Rosenberg et al. [1].]

monophasée correspondant à une valeur de $x$ supérieure à 0,15 . Après une trempe rapide les essais ont donné des matériaux constitués d'une solution solide avec $x=0,15$ au maximum et du tellurure de cadmium comme deuxième phase.

La variation de la largeur de bande interdite $E_{\mathrm{g}}$ avec $x$ est présentée sur la figure 3 où apparaissent aussi les résultats de Nikolic [3]. Pour des valeurs de $x$ supérieures à $0,1,1$ 'absorption très forte des photons, vraisemblablement due aux porteurs libres, n'a pas permis de mettre en évidence le front d'absorption dû aux transitions à travers la bande interdite.

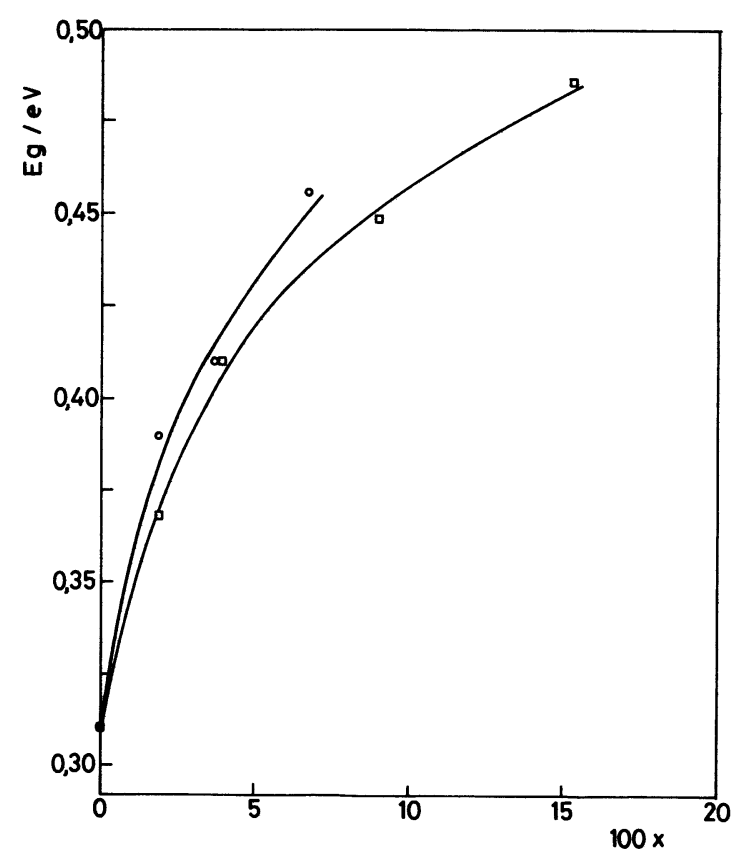

Fig. 3. - Bande interdite en fonction de $x$ des composés $\mathrm{Pb}_{1-x} \mathrm{Cd}_{x} \mathrm{Te}$ : $O$ présents résultats; $\square$ résultats de Nikolic [3].

[Energy gap variation versus $x$ for $\mathrm{Pb}_{1-x} \mathrm{Cd}_{x} \mathrm{Te}$ alloys : $\mathrm{O}$ our results; $\square$ the results of Nikolic [3].] 
Par ailleurs, la plupart des échantillons apparaissent de type $\mathrm{n}$ avec des concentrations supérieures à $10^{18} \mathrm{~cm}^{-3}$, les mobilités à $300 \mathrm{~K}$ varient de 300 à $800 \mathrm{~cm}^{2} \mathrm{~V}^{-1} \mathrm{~s}^{-1}$.

4. Précipitation de CdTe. - A partir des résultats de Rosenberg et al. [1] donnant la variation de $a$ en fonction de $x$ et de ceux de Nikolic [3] donnant la variation de $E_{\mathrm{g}}$ en fonction de $x$, nous avons pu déterminer la variation $E_{\mathrm{g}}(a)$ correspondant à ces travaux, elle est présentée sur la figure 4. Sur cette figure sont

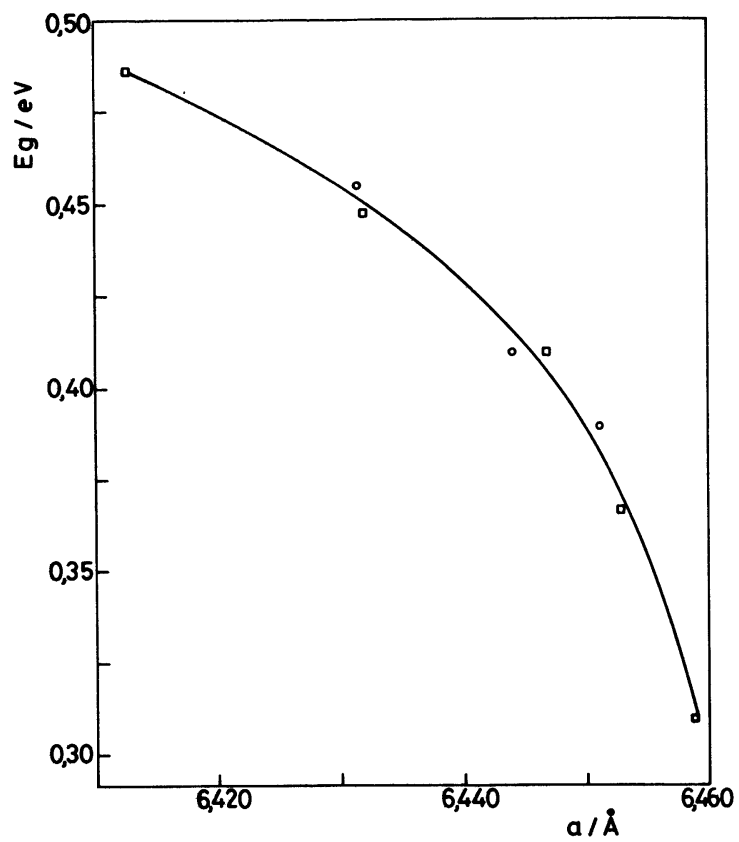

Fig. 4. - Bande interdite en fonction du paramètre de maille des composés $\mathrm{Pb}_{1-x} \mathrm{Cd}_{x} \mathrm{Te}$ : $\bigcirc$ présents résultats; $\square$ résultats de Nikolic et Rosenberg et al. [1, 3].

[Energy gap $E_{\mathrm{g}}$ versus lattice constant $a$ for $\mathrm{Pb}_{1-x} \mathrm{Cd}_{x} \mathrm{Te}$ alloys : O our results; $\square$ The results of Nikolic and Rosenberg et al. [1, 3].]

portés les résultats du présent travail, leur répartition est en bon accord avec la variation $E_{\mathrm{g}}(a)$ tirée de [1] et [3]. On peut donc considérer que l'on a bien affaire à un même matériau $\mathrm{Pb}_{1-x} \mathrm{Cd}_{x} \mathrm{Te}$ mais pour lequel la valeur réelle de $x$ reste à préciser. Le tableau I permet de comparer les valeurs de $c$ correspondant à différents points de $E_{\mathrm{g}}(a)$ indiqués dans [1] et [3] avec celles de nos résultats.

Tableau I

$\begin{array}{cc}\begin{array}{c}\text { Bande } \\ \text { interdite } \\ (\mathrm{eV})\end{array} & \begin{array}{c}\text { Paramètre } \\ \text { de maille } \\ (\AA)\end{array} \\ - & - \\ 0,310 & 6,459 \\ 0,375 & 6,453 \\ 0,419 & 6,443 \\ 0,458 & 6,429 \\ 0,482 & 6,416\end{array}$

Teneur en cadmium $\%$ Références Présents [1] et [3] résultats

$\begin{array}{ccc}\text { maille } & \begin{array}{c}\text { Réferences } \\ {[1] \text { et }[3]}\end{array} & \begin{array}{c}\text { Presents } \\ \text { résultats }\end{array} \\ -459 & - & - \\ 453 & 0 & 0 \\ 443 & 2 & 1,5 \\ 429 & 5 & 3,8 \\ 416 & 10 & 7,4 \\ 4 & 15 & -\end{array}$

Nous pensons que la différence observée vient de l'existence de microprécipités de CdTe qui seraient en quantité plus élevée dans les matériaux étudiés en [1] et [3]. L'existence de précipités a en effet pu être mise en évidence à la microsonde électronique lorsque leur taille est suffisante ce qu'il est possible d'obtenir après un temps de recuit assez long et un refroidissement lent. La valeur $x$ est réduite de la composition globale des échantillons et prend en compte la partie de cadmium présente dans les précipités de CdTe. Il n'est pas possible d'affirmer que les matériaux du présent travail n'ont pas de précipités de CdTe, mais les résultats concernant $a(x)$ et $E(x)$ sont plus près des valeurs réelles.

5. Possibilité d'évaluation de la fraction précipitée par mesure de masse volumique. - La mesure de masse volumique a été utilisée par Rosenberg et al. [2] pour montrer que dans les alliages obtenus les atomes de cadmium se substituent à ceux du plomb alors que leurs matériaux apparaissent contenir une fraction précipitée appréciable (correspondant à plus de $3 \%$ de CdTe précipité pour $x=0,1$ (cf. tableau I)). On peut aussi penser utiliser cette méthode pour évaluer la fraction précipitée.

La précipitation d'une fraction $y$ de CdTe dans la solution solide $\mathrm{Pb}_{1-x} \mathrm{Cd}_{x} \mathrm{Te}$ est traduite par la réaction :

$$
\mathrm{Pb}_{1-x} \mathrm{Cd}_{x} \mathrm{Te} \rightarrow(1-y) \mathrm{Pb}_{1-x^{\prime}} \mathrm{Cd}_{x^{\prime}} \mathrm{Te}+y \mathrm{CdTe}
$$

avec

$$
x^{\prime}=\frac{x-y}{1-y} .
$$

Les masses volumiques $D_{x}$ du matériau sans précipité et $D_{x-y}$ du matériau avec une partie précipitée sont :

$$
D_{x}=\frac{K M}{a_{x}^{3}}, \quad D_{x-y}=\frac{K M}{(1-y) a_{x^{\prime}}^{3}+y a_{\text {CdTe }}^{3}}
$$

où $K$ est une constante,

$M$ est la masse molaire de $\mathrm{Pb}_{1-x} \mathrm{Cd}_{x} \mathrm{Te}$ et $a_{\mathrm{CdTe}}, a_{x}$ et $a_{x^{\prime}}$ sont respectivement les paramètres de maille de $\mathrm{CdTe}$, de la solution solide $\mathrm{Pb}_{1-x} \mathrm{Cd}_{x} \mathrm{Te}$ et de la solution solide $\mathrm{Pb}_{1-x^{\prime}}, \mathrm{Cd}_{x^{\prime}}$ Te. La différence relative de masse volumique est :

$$
\frac{\delta D}{D}=\frac{D_{x-y}-D_{x}}{D_{x-y}}=1-\frac{(1-y) a_{x^{\prime}}^{3}+y a_{\mathrm{CdTe}}^{3}}{a_{x}^{3}}
$$

exprimant $a_{x^{\prime}}$ et $a_{x}$ à partir de la relation (3.1) et développant les expressions au 1 er ordre ce qui est très justifié car $x<0,2$ et $y<0,15$ on trouve, ayant pris $a_{\mathrm{PbTe}}=6,459 \AA$ et $a_{\mathrm{CdTe}}=6,481 \AA$ :

$$
\begin{array}{r}
\frac{\delta D}{D}=-y\left(1,96 \times 10^{-1}+1,34 \times 10^{-1} x-\right. \\
\left.-1,15 \times 10^{-2} y\right) .
\end{array}
$$


La figure 5 représente, par des triangles et pour deux valeurs de $y$, les variations absolues de masse volumique en fonction de $x$ calculées directement. On trouve ainsi que pour $x=0,1$ une précipitation de $4 \%$ de CdTe fait passer la densité de 8,167 à 8,103 ce qui est facilement mis en évidence par mesure de masse volumique standard. Ce calcul ne tient pas compte de la contribution due aux défauts présents dans la matrice de la solution solide.

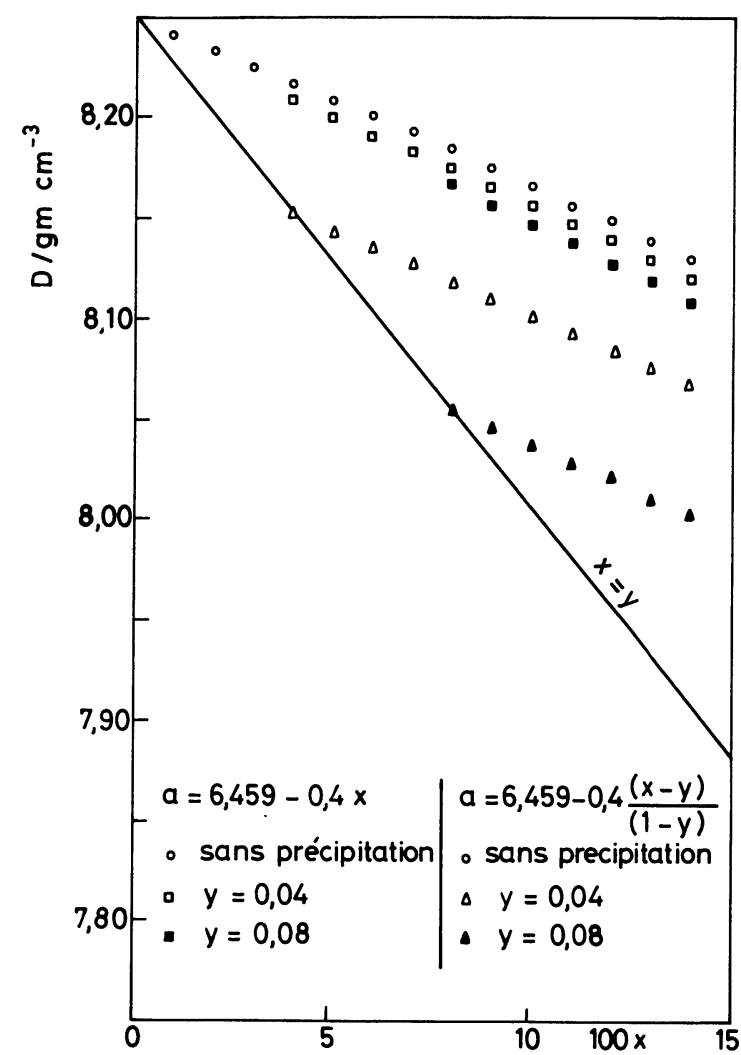

Fig. 5. - Masse volumique des composés en fonction de $x$ pour différentes valeurs de la fraction précipitée $y: \Delta \boldsymbol{\Delta}$ tenant compte de la variation de paramètre de maille; $\square$ sans tenir compte de la variation de paramètre de maille.

[Density of $\mathrm{Pb}_{1-x} \mathrm{Cd}_{x} \mathrm{Te}$ alloys versus $x$ for several fractions of precipitated CdTe : $\triangle \Delta$ taking into account the lattice constant variation. $\square \square$ ignoring the variation of lattice constant.]

La variation de masse volumique, conséquence de la précipitation de $\mathrm{CdTe}$ est principalement due à la variation du paramètre de maille (terme $y 1,96 \times 10^{-1}$ prépondérant). Pour savoir si une solution solide obtenue par trempe contient une fraction précipitée appréciable il faut comparer sa masse volumique avec celle que l'on peut calculer à partir de la mesure du paramètre de maille ou de sa valeur tirée de la relation (3.1) à partir de la connaissance de la composition totale $x$ en cadmium. On est alors amené à comparer la masse volumique d'un échantillon sans précipité avec celle du même échantillon contenant une fraction $y$ de précipité et de même paramètre de maille puisque donné par la mesure directe ou déduite de la valeur $x$. Il faut refaire le calcul précédent mais sans changer le paramètre de maille car seul, celui donné par l'expérience est accessible. Les masses volumiques à comparer sont $D_{x}$ donné par (5.2) d'une part et

$$
D_{x-y}^{\prime}=\frac{K M}{(1-y) a_{x}^{3}+y a_{\mathrm{CdTe}}^{3}}
$$

d'autre part, soit en variation relative et numériquement

$$
\frac{\delta D^{\prime}}{D^{\prime}}=-y\left(1,02 \times 10^{-2}+0,188 x\right) .
$$

Les variations absolues de masse volumique en fonction de $x$ ont été représentées par des carrés sur la figure 5 pour des matériaux ayant $4 \%$ et $8 \%$ de CdTe précipités. On remarque que l'existence dans une solution solide ayant $x=0,1$, de $4 \%$ de cadmium précipité en CdTe donne un écart de masse volumique par rapport à la solution solide sans précipité de 0,01 soit un écart relatif de $1,2 \times 10^{-3}$ qui ne peut être mis en évidence avec des appareillages standards de mesure de masse volumique. De plus, un calcul simple montre que l'écart de masse volumique correspondant à une fraction $y=1 \%$ précipitée est le même que celui résultant d'une densité de lacunes de plomb de $10^{19} \mathrm{~cm}^{-3}$ qui se rencontre dans ces types de matériaux $[9,10]$. Il n'est donc pas possible d'affirmer à partir de mesures de masse volumique que les échantillons présentés ici n'ont pas encore une fraction précipitée de $\mathrm{CdTe}$ de quelques \% bien que ceci n'ait pu être mis en évidence par d'autres méthodes. Il apparaît par contre, que les résultats donnés ici concernant $a(x)$ et $E_{\mathrm{g}}(x)$ sont plus proches des valeurs réelles que ceux donnés précédemment.

Remerciements. - Nous remercions Monsieur Triboulet (Laboratoire de Physique des Solides, C.N.R.S. Bellevue) pour la fourniture de CdTe et son assistance efficace dans l'élaboration des matériaux.

\section{Bibliographie}

[1] Rosenberg, A. J., Grierson, R., Woolley, J. C. and NikoLIC, P. M., Tr. Metall. Soc., A.I.M.E. 230 (1964) 342.

[2] Rosenberg, A. J. and Wald, F., J. Phys. Chem. Solids 26 (1965) 1079 .

[3] Nikolic, P. M., Br. J. Appl. Phys. 17 (1966) 341.

[4] Rogers, L. M. and Crocker, A. J., J. Phys. D, 4 (1971) 1006.

[5] Averkin, A. A., Gurieva, E. A., Efimova, B. A. and Stil' BANS, L. S. Fiz. Tekh. Poluprovodn 12 (1978) 1144 (Sov. Phys. Semicond. 12 (1978) 678).

[6] Veis, A. N. and Nemov, S. A., Fiz. Tekh. Poluprovodn 13 (1979) 1661 (Sov. Phys. Semicond. 13 (1979) 969).
[7] CdTe fourni par R. Triboulet, Lab. de Physique des Solides C.N.R.S. Bellevue.

[8] La plus grande partie des mesures a été faite à la microsonde Ouest (Brest) et au Laboratoire du C.N.R.S. Bellevue.

[9] Brebrick, R. F., in Progress in Solid State Chemistry, H. Reiss, (Pergamon Press, Oxford) 1966, p. 213.

[10] Novoselova, A. V., Matveyev, V. P. and Gaskov, A. M., Progress in solid State Chemistry, H. Reiss and J. O. McCaldin (Pergamon Press, New York) 1972, vol. 7. 